\title{
Hax-1 is rapidly degraded by the proteasome dependent on its PEST sequence
}

\author{
Bin $\mathrm{Li}^{1,2}$, Qingsong Hu${ }^{1,2}$, Ranjie $\mathrm{Xu}^{1,2}$, Haigang Ren ${ }^{1}$, Erkang Fei ${ }^{2}$, Dong Chen ${ }^{1}$ and Guanghui Wang ${ }^{1 *}$
}

\begin{abstract}
Background: HS-1-associated protein X-1 (Hax-1), is a multifunctional protein that has sequence homology to $\mathrm{BCl}-2$ family members. HAX-1 knockout animals reveal that it plays an essential protective role in the central nervous system against various stresses. Homozygous mutations in the HAX-1 gene are associated with autosomal recessive forms of severe congenital neutropenia along with neurological symptoms. The protein level of Hax-1 has been shown to be regulated by cellular protease cleavage or by transcriptional suppression upon stimulation.

Results: Here, we report a novel post-translational mechanism for regulation of Hax-1 levels in mammalian cells. We identified that PEST sequence, a sequence rich in proline, glutamic acid, serine and threonine, is responsible for its poly-ubiquitination and rapid degradation. Hax-1 is conjugated by K48-linked ubiquitin chains and undergoes a fast turnover by the proteasome system. A deletion mutant of Hax-1 that lacks the PEST sequence is more resistant to the proteasomal degradation and exerts more protective effects against apoptotic stimuli than wild type Hax-1.
\end{abstract}

Conclusion: Our data indicate that Hax-1 is a short-lived protein and that its PEST sequence dependent fast degradation by the proteasome may contribute to the rapid cellular responses upon different stimulations.

Keywords: Hax-1, Proteasome, Ubiquitin, PEST sequence, Bcl-2 family protein

\section{Background}

HS-1-associated protein $\mathrm{X}-1$, Hax-1, is a $35 \mathrm{kDa}$ protein with two Bcl-2 homology $(\mathrm{BH})$ domains that was identified in a yeast two hybrid screen where it was found to interact with HS-1, a Src kinase substrate [1]. Hax-1 is ubiquitously expressed in most tissues and is reported to be localized in mitochondria as well as the endoplasmic reticulum (ER) and nuclear membrane [1-3]. Mutations identified in the human $H A X-1$ gene have been shown to cause neutropenia and neurodevelopmental abnormalities [4-6]. Knockout $H A X-1$ mice show increased apoptosis of neurons and postnatal lethality. [7]. Hax-1 is a multifunctional protein that plays roles in calcium homeostasis [8], cell migration [9] and apoptotic regulation [10,11]. It was reported that Hax-1 protects cells against various stimuli and has been shown to interact with a number of cellular

\footnotetext{
* Correspondence: wanggh@suda.edu.cn

'Laboratory of Molecular Neuropathology, Department of Pharmacology, Soochow University College of Pharmaceutical Sciences, Suzhou, Jiangsu 201203, People's Republic of China

Full list of author information is available at the end of the article
}

and viral proteins to suppress their pro-death properties [12-15]. In addition, Hax-1 has been found to be up-regulated in breast cancer, lung cancer and melanoma [16], suggesting that it also has a role in oncogenesis.

A PEST sequence is a peptide sequence which is rich in proline $(\mathrm{P})$, glutamic acid $(\mathrm{E})$, serine $(\mathrm{S})$, and threonine $(\mathrm{T})$. It is known that the PEST sequence functions as a proteolytic signal to target proteins for degradation resulting in short intracellular half lives [17]. For example, the PEST sequence of NF-kappa B is responsible for its cleavage by calpain [18]. It was reported that c-myc, a protein with a PEST sequence, has a half-life shorter than one hour [17]. Notch 1, another short-lived protein, is ubiquitinated by an E3 ligase sel-10 and degraded by the proteasome dependent on its PEST sequence $[19,20]$.

Hax-1 was predicted to contain a PEST sequence (aa 104-117) [1], however, it is still unknown whether this PEST sequence effects its turnover rate. In this study, we investigated the stability of Hax-1 in different cells and explored the role of the PEST sequence in its degradation and biological function. 


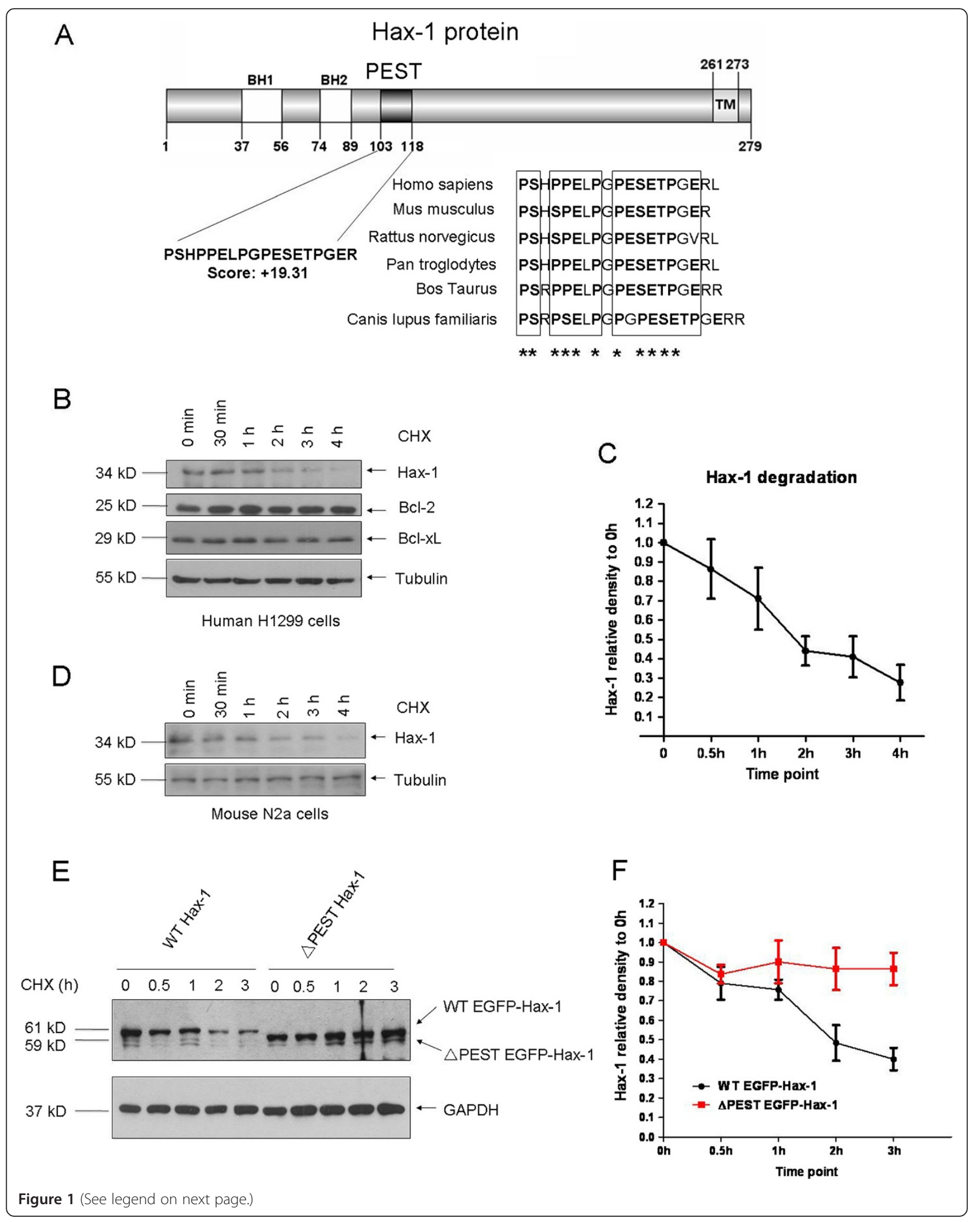


(See figure on previous page.)

Figure 1 Rapid degradation of Hax-1 is dependent on its PEST sequence. A. Schematic representation of a PEST sequence in Hax-1 protein. The PEST sequence was identified using Pestfind service on "emboss.bioinformatics.nl/cgi-bin/emboss/pestfind". The PEST sequence in Hax-1 is conserved among different mammals. B. Chase-time experiment of Hax-1 and other Bcl-2 proteins. H1299 cells treated with CHX (100 ug/ml) for different time points were harvested for immunoblot analysis using indicated antibodies. C. Data from three independent experiments in B were quantified. D. Similar experiments as B were carried out using mouse N2a cells. E. An EGFP-tagged WT Hax-1 or $\triangle$ PEST Hax-1 was transiently transfected into H1299 cells. Forty-eight hours later, CHX chase experiments were carried out. F. Quantitative analysis of data from E with three independent experiments.

\section{Results}

\section{Rapid degradation of Hax-1}

In addition to its $\mathrm{BH}$ domains and a trans-membrane domain, Hax-1 has a PEST sequence [1]. The PEST region in Hax-1 is highly conserved in mammalian animals (Figure 1A). We tested the degradation profile of Hax-1 using a cycloheximide ( $\mathrm{CHX}$ ) chase experiment in both human lung cancer cell line H1299 and mouse neuroblastoma cell line N2a. Hax-1 was found to have a much shorter half-life than other two pro-survival Bcl-2 family proteins, Bcl-2 and Bcl-xL (Figure 1B-D), suggesting that the Hax-1 protein is unstable and is rapidly degraded.

\section{PEST sequence-dependent degradation of Hax-1}

We next tested whether the PEST sequence in Hax-1 is responsible for its rapid degradation. A deletion mutant of Hax-1 was constructed in which the PEST sequence (aa 103-118) was deleted. The CHX chase experiments showed that the $\triangle$ PEST Hax-1 level remained largely unchanged up to 3 hours, whereas WT Hax-1 level rapidly decreased to $<50 \%$ within 3 hours (Figure $1 \mathrm{E}$ and $\mathrm{F})$, suggesting that the PEST sequence in Hax-1 is necessary for its rapid degradation.

\section{Degradation of Hax-1 by the ubiquitin-proteasome pathway}

Proteasome and autophagy systems are two main pathways for protein degradation. Here we tested which pathway is involved in the fast-turnover of Hax-1. Cells were treated with MG132, a proteasome inhibitor, or Bafilomycin A1, an autophagy inhibitor. The level of EGFP-Hax-1 increased in cells treated with MG132 for 3 hours (Figure 2A), whereas in cells treated with Bafilomycin A1 the protein level remained unchanged up to 18 hours (Figure 2B). These data suggest that Hax-1 is mainly degraded by the proteasome, but not by autophagy-lysosome pathway. A time-dependent increase in endogenous Hax-1 level was also observed in cells treated with MG132 (Figure 2C). We next examined the turnover of endogenous Hax-1 in the presence of MG132 using CHX chase experiments. In the presence of MG132, endogenous Hax-1 was not observed to be degraded within 4 hours, however, in the absence of MG132, it was rapidly degraded after two hours (Figure 2D).

\section{Hax-1 conjugation with K48-linked ubiquitin chains is dependent on the PEST sequence}

We have shown that Hax-1 is degraded by the proteasome. Usually, the proteasomal degradation process requires polyubiquitination of the substrates [21]. We therefore tested if Hax-1 is ubiquitinated and if yes, what kind of ubiquitin conjugation is involved in the degradation of Hax-1. Enhanced ubiquitination of Hax-1 was observed in the presence of MG132 than that in the absence of MG132 (Figure 3A) as revealed by co-immunoprecipitation experiments. Then, we examined the polyubiquitin of Hax-1 with two specific antibodies which recognize K48- or K63-linked ubiquitin, respectively. Increased polyubiquitination of Hax-1 was detected with an antibody specific to K48-linked polyubiquitin, but not with that to K63-linked polyubiquitin (Figure 3B), suggesting that Hax-1 is mainly conjugated by the K48-linked ubiquitin chains. We next evaluated if the PEST sequence affects Hax-1 polyubiquitination. We found that the deletion of the PEST sequence in Hax-1 greatly decreased its polyubiquitination (Figure $3 \mathrm{C}$ ), suggesting that the PEST sequence in Hax-1 is necessary for its ubiquitination.

\section{Increased degradation of Hax-1 during apoptosis}

As Hax-1 is known to be an anti-apoptotic protein, we hypothesized whether its degradation is regulated under apoptosis. We transfected H1299 cells with EGFP-Hax-1 and treated them with DMSO or staurosporine (STS), an inducer of apoptosis. In the absence of MG132, the amounts of Hax-1 protein decreased with increasing concentration of STS, however, in the presence of MG132, the trend was largely attenuated (Figure 3D and E), suggesting an accelerated degradation of Hax-1 by the proteasome under apoptosis.

\section{$\triangle$ PEST Hax-1 mutant attenuated STS-induced cell death}

As overexpression of Hax-1 has been shown to have an anti-apoptotic effect and also regulates mitochondria membrane potential [10], we examined the effects of knockdown of Hax-1 on STS-induced apoptosis. The efficacy of the siRNA against Hax-1 was evaluated (Figure 4A). STS induced significantly higher level of apoptosis in those cells in which Hax-1 levels were knocked down as compared to control cells. This 


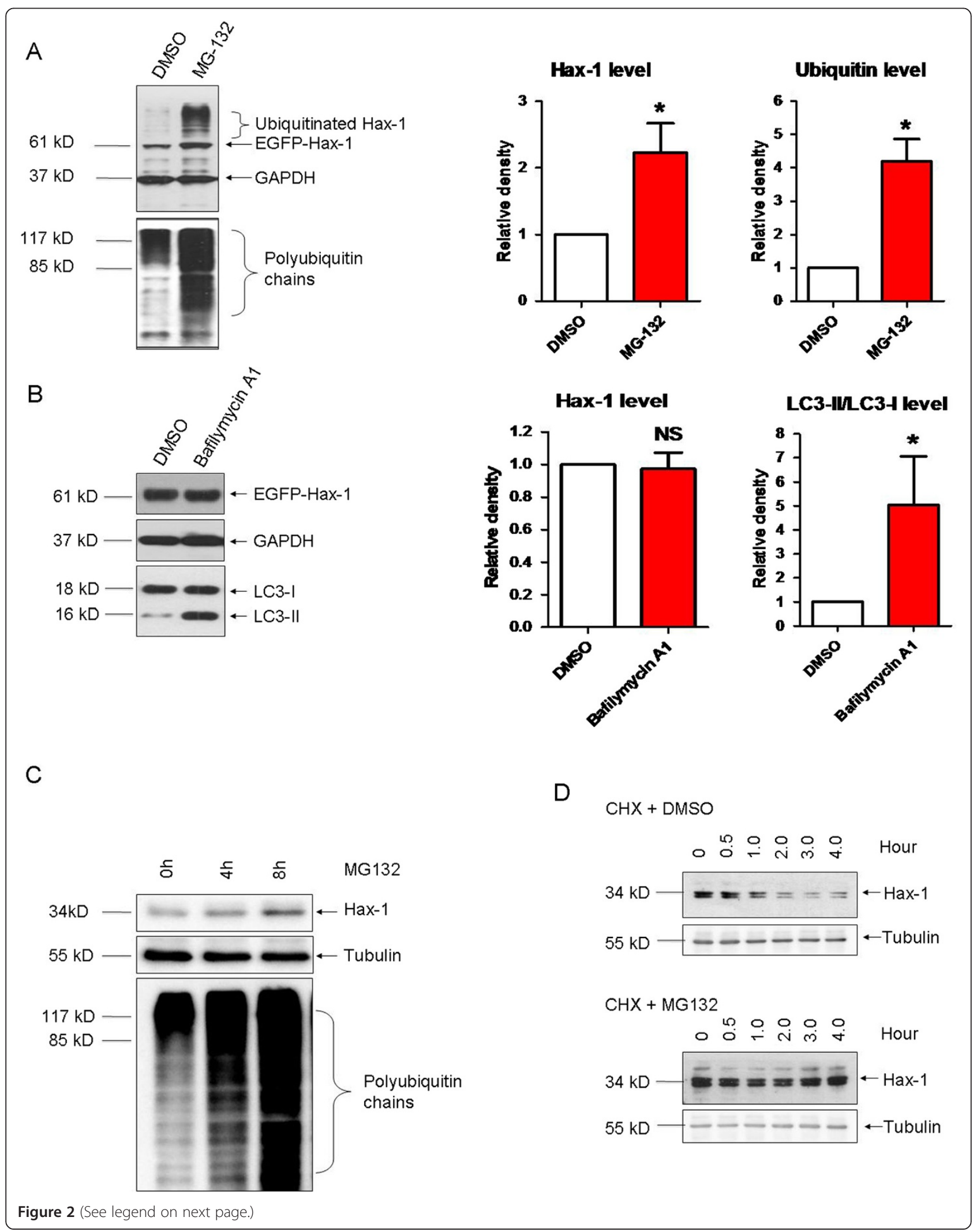


(See figure on previous page.)

Figure 2 Proteasomal degradation of Hax-1. A and B. H1299 cells were transiently transfected with EGFP-Hax-1. Forty-eight hours later, cells were treated with MG132 (1 $\mu \mathrm{M})$ for 3 hours (A) or Bafilomycin A1 (10 nM) for 18 hours (B). Cells were then harvested and immunoblotted using anti-GFP, ubiquitin, LC3 or GAPDH antibody. Detections of polyubiquitin (A, left, lower panel) and LC3-II (B, left, lower panel) levels in input were used to indicate polyubiquitination levels (A) and autophagy levels (B). Data from three independent experiments were quantified (means \pm S.E.M., ${ }^{*} p<0.05$, one-way ANOVA). C. Time dependent increase of Hax-1 levels by the proteasomal inhibition (Upper panel). Increased levels of total ubiquitin were served as positive controls for MG132 treatment. Tubulin was served as loading control. D. H1299 cells were treated with CHX with or without MG132 for chase experiments.

increase in apoptosis also elevated with increased STS dosage (Figure 4B). Using JC-1 (a lipophilic, cationic dye that selectively enters into mitochondria and reversibly changes its color from green to red as the membrane potential increases) staining, we found that mitochondrial potential was also greatly decreased in Hax-1 knockdown cells than in control cells upon CCCP (Carbonyl cyanide $\mathrm{m}$-chlorophenyl hydrazone, an inducer to cause mitochondrial permeability transition) treatment (Figure 4C). These data indicate that Hax-1 is important for cells against apoptotic stress or mitochondrial damage. We next transfected cells with WT Hax-1 or $\triangle \mathrm{PEST}$ Hax-1 and then treated cells with STS. Fewer condensed nuclei were observed in EGFP- $\triangle$ PEST Hax-1 expressing cells than in EGFP-Hax-1 expressing cells (Figure 4D and E), suggesting that deletion of PEST sequence may increase Hax-1 stability, causing more resistance to STSinduced apoptosis.

\section{Discussion}

Hax-1 transcript levels in mouse kidney, testis, and liver have previously been found to not directly correlate with detected protein levels [22]. Similar phenomenon has also been observed in rat tissues [23]. Two hypotheses to explain the different levels of mRNA compared to protein are that either high amounts of the Hax-1 transcript do not translate into proteins or that the protein degradation rate of Hax-1 is considerably high [24]. Here, we provide clear evidence showing that Hax-1 protein is indeed turned over at a fast rate in a proteosome dependent manner. It is important to note that, Hax-1 exists as many as 7 alternative splicing forms $[16,23]$, and these splicing variants may play important roles in development or tumor formation. For example, the internal deletions in variants vII, vIV and vVI result in removal of $\mathrm{BH}$ domains and changes in PEST domain from variants I (the full length 278aa form which is investigated in this paper) [23]. It is therefore possible that these variant forms of Hax-1, because of its impairment in PEST degradation signal, is more stable than its dominant form variant $I$. The population of cells bearing an up-regulation of these variants shows enhanced protective roles in tissues or more oncogenic activity, as evidenced in tumors [16].
Polyubiquitination is required for the protein degradation by the proteasome [21]. Ubiquitin molecules, which form ubiquitin chains to a protein, are covalently linked to each other between a lysine site (K11, K29, K48 or K63) of the previous ubiquitin and the carboxyterminal glycine of a new ubiquitin. K48-linked polyubiquitination of a protein usually mediates its degradation by the proteasome, however, K63-linked polyubiquitination is most likely to play roles in translation, endocytosis and other functions [25-27]. In the present report, we demonstrate that Hax-1 is ubiquitinated via K48linked ubiquitin chains. The ubiquitination of Hax-1 is largely dependent on its PEST sequence. In many shortlived proteins, the PEST sequence serves as a signal sequence to drive their proteolysis or rapid degradation [17]. In some cases, ubiquitination of proteins depends upon their PEST sequence [19]. Here, we found that deletion of the PEST sequence results in much less ubiquitination of Hax-1, thereby increasing its stability. It is therefore possible that the PEST sequence in Hax-1 is responsible for its proper folding to be conjugated with the ubiquitin chains. The PEST sequence is also reported to be a motif that is involved in protein modification. For example, phosphorylation of a PEST sequence by casein kinase II (CKII) appears to promote the degradation of IкB $\alpha$ [28]. Also, a PEST-like sequence has been shown to mediate phosphorylation and efficient ubiquitination of yeast uracil permease [29]. Further studies to identify if the PEST sequence in Hax-1 is phosphorylated and if this modification affects Hax-1 stability will be of help to explore the exact role of the PEST sequence in Hax-1.

Hax-1 is structurally similar to $\mathrm{Bcl}-2$ for its $\mathrm{BH}$ domains and TM domain. However, Hax-1 is less stable compared to other Bcl-2 family proteins [30,31]. It was reported that Hax-1 is rapidly cleaved by caspase 3 [32], HtrA2 [10] or Granzyme B [33] during cell death. It is therefore possible that these enzymes contribute to Hax-1 degradation in apoptosis. As Hax-1 is a shortlived protein and also degraded by the proteasome, it suggests that the proteasomal degradation of Hax-1 highly regulates Hax-1 levels in normal conditions. Knockdown of pleiotropic human prohibitin 2 in HeLa cells results in caspase-dependent apoptosis through down-regulation of Hax-1 [34]. Here, we report that, in addition to protease cleavage, the proteasomal degradation is also an important post-translational regulation 


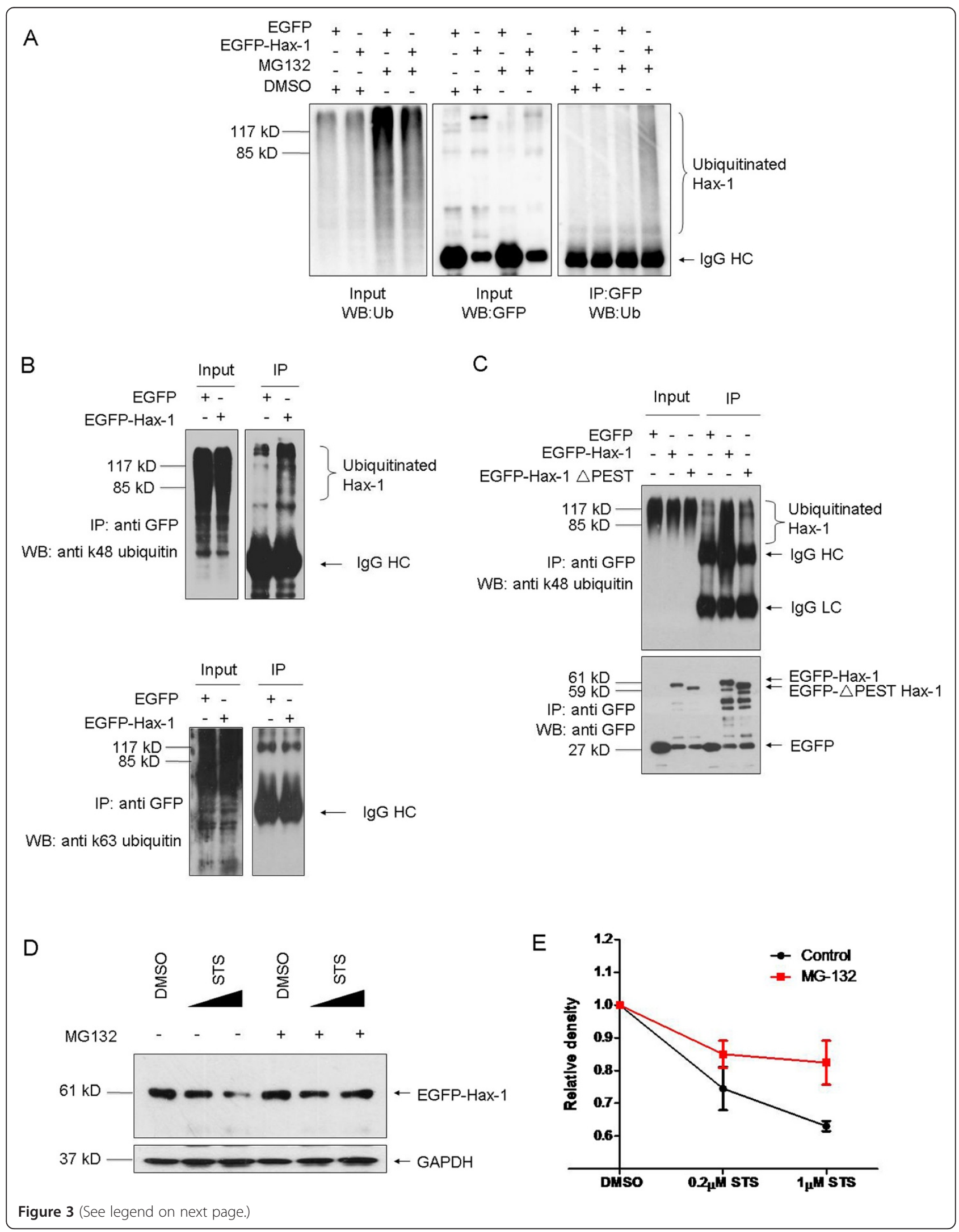


(See figure on previous page.)

Figure 3 K48-linked ubiquitiniation of Hax-1 enhanced proteasomal degradation of Hax-1 during apoptosis. A. H1299 cells were transfected with EGFP or EGFP-Hax-1. Forty-eight hours later, cells were treated with or without MG132 (1 $\mu$ M) for 12 hours. Cell lysates were then subjected to immunoprecipitation with anti-GFP antibodies and the immunoprecipitants were detected with total ubiquitin antibody. B. Similar experiments as A were performed using specific anti-K48 or -K63 ubiquitin antibodies. C. H1299 cells were transfected with EGFP or EGFP-Hax-1 or EGFP- $\triangle$ PEST Hax-1 expression constructs. Forty-eight hours later, cells were treated with MG132 $(1 \mu \mathrm{M})$ for 12 hours. Cell lysates were then immunoprecipitated with anti-GFP antibodies and detected with anti-K48 ubiquitin antibodies. D. EGFP-Hax-1 transfected H1299 cells were treated with DMSO or increased levels of STS (0.2-1 umol) in the absence or presence of MG132 for 3 hours. Cells were then harvested and subjected to immunoblot analysis with anti-GFP antibody. E. Quantitative analyses of Hax-1 degradation upon STS treatment through three independent experiments were shown.

for Hax-1 during apoptosis (Figure 3D and E). When the PEST sequence is abolished, Hax-1 is shown to convey increased resistance to cell death. Taken together, these data suggest that Hax-1 may be rapidly subjected to proteolysis in response to cellular stresses, resulting in a decrease in its protein level and hence loss of its protective activity.

\section{Conclusions}

In summary, our study demonstrates that Hax-1 is rapidly degraded by the proteasome in a PEST sequence dependent manner. During apoptosis, degradation of Hax-1 is enhanced whereas expression of $\triangle$ PEST mutant of Hax-1 protects cells against apoptotic stimulation.

\section{Methods}

\section{Cell culture, transfections and drug treatments}

N2a and H1299 cells were grown in Dulbecco's Modified Eagle's Medium (DMEM, GIBCO) containing $10 \%$ fetal calf serum with $100 \mu \mathrm{g} / \mathrm{ml}$ penicillin and $100 \mu \mathrm{g} / \mathrm{ml}$ streptomycin. Transfections were performed using Lipofectamine 2000 (Invitrogen) according to the manufacturer's instructions. In order to ensure equal transfection efficiency, master mix of the same plasmids were made and aliquot to each well, we double check the equal expression of EGFP-Hax-1 through fluoresce microscopy before drug treatment (by deem to lowest exposure). Hoechst 33342, DAPI, STS (Staurosporine), Bafilomycin A1, Annexin V, PI (Propidium iodide) and $\mathrm{CHX}$ (cycloheximide) were purchased from Sigma. MG132 was obtained from Calbiochem.

\section{Plasmids}

The Hax-1 related constructs were described previously [35]. A PEST sequence deletion mutant was created using the following primers: 5'-ACCAAGATCACTAAACCA-3' and 5'-CTGTAGAACCGGGCCAAG-3'.

\section{siRNAs}

35 pmoles of each siRNA were transfected using Oligofectamine, according to the manufacturer's instructions (Invitrogen). Oligonucleotides were purchased from
GenePharma (Shanghai, China) and had the following sequences:

$$
\begin{aligned}
& \text { si } \operatorname{Hax} \text {-1 sense: } \\
& \text { 5'-AACCAGAGAGGACAAUGAUCUdTdT-3'. } \\
& \text { si } \operatorname{Hax} \text {-1 antisense: } \\
& \text { 5'-AGAUCAUUGUCCUCUCUGGUUdTdT-3'. } \\
& \text { si Control sense: } \\
& \text { 5'-UUCUCCGAACGUGUCACGUdTdT-3'. } \\
& \text { si Control antisense: } \\
& \text { 5'-ACGUGACACGUUCGGAGAAdTdT-3'. }
\end{aligned}
$$

\section{Immunoblot analysis and antibodies}

Cell extracts were lysed in $1 \times$ RIPA lysis buffer (25 mM Tris- $\mathrm{HCl}, \mathrm{pH} 7.6,150 \mathrm{mM} \mathrm{NaCl}, 1$ \% NP-40 and $1 \%$ sodium deoxycholate) in the presence of protease inhibitor cocktail (Roche). Approximately $20 \mu \mathrm{g}$ of cell lysates was separated on SDS-PAGE and transferred onto a PVDF membrane (Millipore). Immunoblot analyses were carried out with the following primary antibodies: anti-Bcl-2 (Abcam), anti-Bcl-xL (Cell Signaling Technology), anti-GAPDH (Chemicon), anti-GFP (Santa Cruz Biotechnology), anti-LC3 (Novas), anti-Tubulin (Merck Chemicals), anti-Hax-1 (BD Biosciences), anti-Flag (Sigma), anti-ubiquitin (Santa Cruz Biotechnology), anti-K48-ubiquitin (Millipore) and anti-K63-ubiquitin (Millipore). The secondary antibodies, i.e., sheep anti-mouse IgG-HRP or anti-rabbit IgG-HRP, were from Amersham Pharmacia Biotech. The proteins were visualized using an ECL detection kit (Amersham Pharmacia Biotech).

\section{Immunoprecipitation}

Cells transfected with the indicated plasmids were collected $48 \mathrm{hrs}$ after transfection and were lysed in TSPI buffer containing $50 \mathrm{mM}$ Tris- $\mathrm{HCl}, \mathrm{pH} 7.5,150 \mathrm{mM}$ sodium chloride, $1 \mathrm{mM}$ EDTA and $1 \% \mathrm{NP}-40$ supplemented with complete mini protease inhibitor cocktail (Roche). Cellular debris was removed by centrifugation at $12,000 \mathrm{~g}$ for 30 minutes at $4^{\circ} \mathrm{C}$. The supernatants were incubated with anti-GFP antibodies overnight at $4^{\circ} \mathrm{C}$. After incubation, protein G Sepharose (Roche) was used for precipitation. The beads were washed with TSPI 


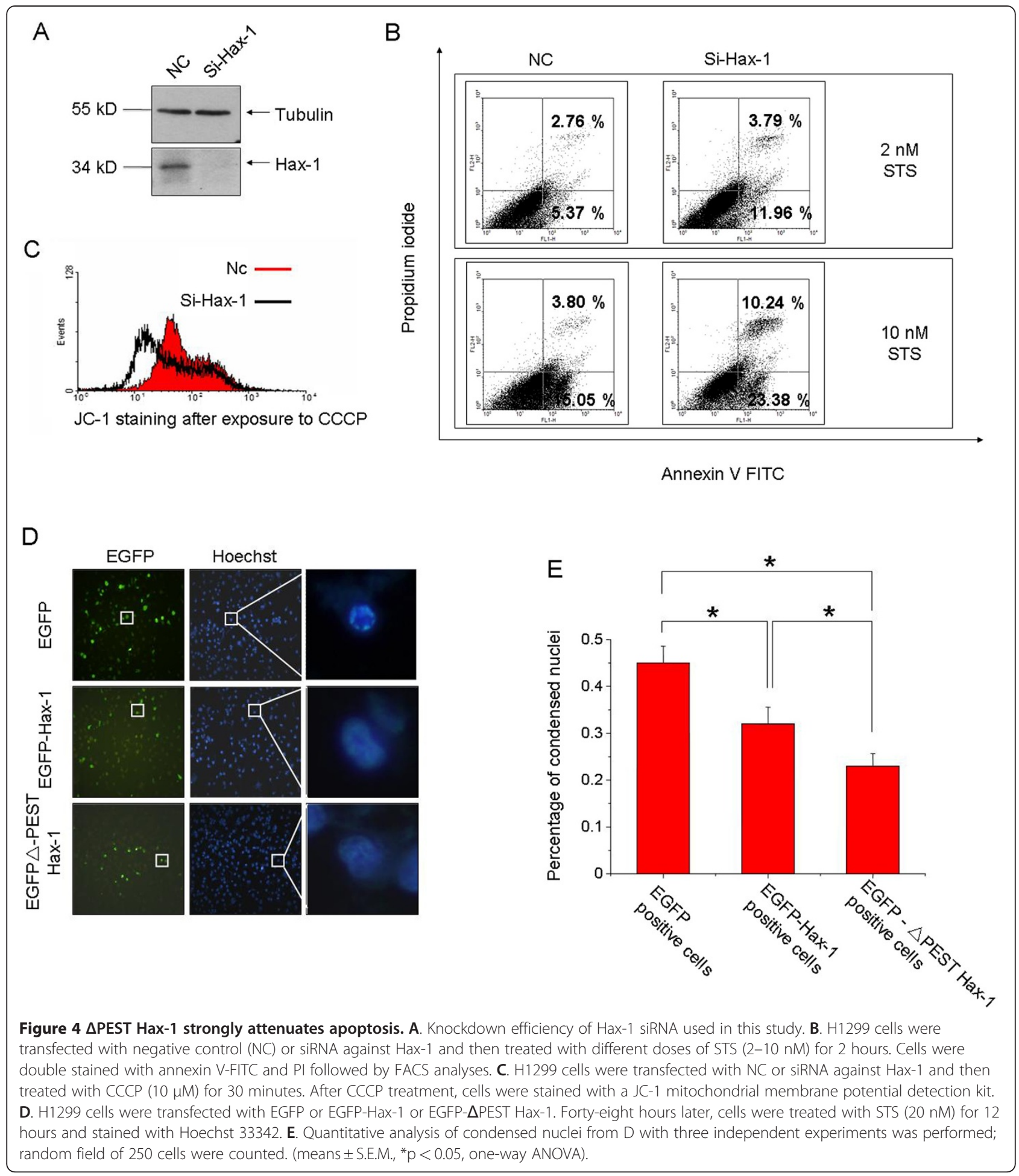

buffer four times and then eluted with SDS sample buffer for immunoblot analysis.

\section{Statistical analysis}

Densitometric analysis of immunoblots from three independent experiments was performed using ImageJ windows version. The data were analyzed using windows version of Origin 6.0 (Originlab) or Prism 5 (Graphpad softwere). The pictures in Figure 1A were draw using DOG 1.0 [36].

\section{Abbreviations}

CHX: Cycloheximide; Hax-1: HS-1-associated protein X-1; PCR: Polymerase Chain Reaction; SDS-PAGE: Sodium Dodecyl Sulfate-Polyacrylamide Gel 
Electrophoresis; siRNA: Small interfering RNA; STS: Staurosporine; CCCP: Carbonyl Cyanide m-Chlorophenyl hydrazone.

\section{Competing interests}

The authors declare no conflict of interest in this paper

\section{Authors' contributions}

BL performed most experiments in plasmid construction, immunoblot analyses, immunoprecipitations, cell culture, transfections, drug treatments, siRNAs, statistical analysis and drafted the manuscript; QH performed parts of experiments in cell transfections, immunoprecipitationss; RX performed part of plasmid construction; HR performed part of cell culture and transfections; EF performed part of plasmid construction; DC performed part of cell cultures; GW conceived of the study, participated in its design and coordination and revised manuscript. All authors read and approved the final manuscript.

\section{Acknowledgements}

This work was supported in part by the grants from the National High-tech Research and Development program of China 973-projects (2012CB947602), a Project Funded by the Priority Academic Program Development of Jiangsu Higher Education Institutions, and the National Natural Sciences Foundation of China (Nos. 30970921 and 30900412).

\section{Author details}

'Laboratory of Molecular Neuropathology, Department of Pharmacology, Soochow University College of Pharmaceutical Sciences, Suzhou, Jiangsu 201203, People's Republic of China. ${ }^{2}$ Laboratory of Molecular Neuropathology and Key Laboratory of Brain Function and Diseases, School of Life Sciences, University of Science \& Technology of China, Chinese Academy of Sciences, Hefei, Anhui 230027, People's Republic of China.

\section{Received: 5 March 2012 Accepted: 13 July 2012}

Published: 24 July 2012

\section{References}

1. Suzuki Y, Demoliere C, Kitamura D, Takeshita H, Deuschle U, Watanabe T: HAX-1, a novel intracellular protein, localized on mitochondria, directly associates with $\mathrm{HS} 1$, a substrate of Src family tyrosine kinases.J Immuno 1997, 158(6):2736-2744.

2. Gallagher AR, Cedzich A, Gretz N, Somlo S, Witzgall R: The polycystic kidney disease protein PKD2 interacts with Hax-1, a protein associated with the actin cytoskeleton.Proc Natl Acad Sci U S A 2000, 97(8):4017-4022.

3. Kawaguchi Y, Nakajima K, Igarashi M, Morita T, Tanaka M, Suzuki M, Yokoyama A, Matsuda G, Kato K, Kanamori M, et al: Interaction of EpsteinBarr virus nuclear antigen leader protein (EBNA-LP) with HS1-associated protein X-1: implication of cytoplasmic function of EBNA-LP.J Virol 2000, 74(21):10104-10111.

4. Ishikawa N, Okada S, Miki M, Shirao K, Kihara H, Tsumura M, Nakamura K, Kawaguchi H, Ohtsubo M, Yasunaga S, et al: Neurodevelopmental abnormalities associated with severe congenital neutropenia due to the R86X mutation in the HAX1 gene.J Med Genet 2008, 45(12):802-807.

5. Klein C, Grudzien M, Appaswamy G, Germeshausen M, Sandrock I, Schaffer AA, Rathinam C, Boztug K, Schwinzer B, Rezaei N, et al: HAX1 deficiency causes autosomal recessive severe congenital neutropenia (Kostmann disease).Nat Genet 2007, 39(1):86-92.

6. Rezaei N, Chavoshzadeh Z, Alaei OR, Sandrock I, Klein C: Association of HAX1 deficiency with neurological disorder.Neuropediatrics 2007, 38(5):261-263.

7. Chao JR, Parganas E, Boyd K, Hong CY, Opferman JT, Ihle JN: Hax1mediated processing of $\mathrm{HtrA2}$ by Parl allows survival of lymphocytes and neurons. Nature 2008, 452(7183):98-102.

8. Zhao W, Waggoner JR, Zhang ZG, Lam CK, Han P, Qian J, Schroder PM, Mitton B, Kontrogianni-Konstantopoulos A, Robia SL, Kranias EG: The antiapoptotic protein HAX-1 is a regulator of cardiac function.Proc Natl Acad Sci U S A 2009, 106(49):20776-20781.

9. Radhika V, Onesime D, Ha JH, Dhanasekaran N: Galpha13 stimulates cell migration through cortactin-interacting protein Hax-1.J Biol Chem 2004, 279(47):49406-49413.

10. Cilenti L, Soundarapandian MM, Kyriazis GA, Stratico V, Singh S, Gupta S, Bonventre JV, Alnemri ES, Zervos AS: Regulation of HAX-1 anti-apoptotic protein by Omi/HtrA2 protease during cell death J Biol Chem 2004, 279(48):50295-50301.

11. Vafiadaki E, Sanoudou D, Arvanitis DA, Catino DH, Kranias EG, KontrogianniKonstantopoulos A: Phospholamban interacts with HAX-1, a mitochondrial protein with anti-apoptotic function.J Mol Biol 2007, 367(1):65-79.

12. Matsuda G, Nakajima K, Kawaguchi Y, Yamanashi Y, Hirai K: Epstein-Barr virus (EBV) nuclear antigen leader protein (EBNA-LP) forms complexes with a cellular anti-apoptosis protein $\mathrm{BCl}-2$ or its $\mathrm{EBV}$ counterpart BHRF1 through HS1-associated protein X-1.Microbiol Immunol 2003, 47(1):91-99.

13. Han Y, Chen YS, Liu Z, Bodyak N, Rigor D, Bisping E, Pu WT, Kang PM: Overexpression of HAX-1 protects cardiac myocytes from apoptosis through caspase-9 inhibition. Circ Res 2006, 99(4):415-423.

14. Modem S, Reddy TR: An anti-apoptotic protein, Hax-1, inhibits the HIV-1 rev function by altering its sub-cellular localization. J Cell Physiol 2008, 214(1):14-19.

15. Kang YJ, Jang M, Park YK, Kang S, Bae KH, Cho S, Lee CK, Park BC, Chi SW, Park SG: Molecular interaction between HAX-1 and XIAP inhibits apoptosis.Biochem Biophys Res Commun 2010, 393(4):794-799.

16. Trebinska A, Rembiszewska A, Ciosek K, Ptaszynski K, Rowinski S, Kupryjanczyk J, Siedlecki JA, Grzybowska EA: HAX-1 overexpression, splicing and cellular localization in tumors.BMC Cancer 2010, 10:76.

17. Rogers $S$, Wells $R$, Rechsteiner M: Amino acid sequences common to rapidly degraded proteins: the PEST hypothesis.Science 1986, 234(4774):364-368.

18. Shumway SD, Maki M, Miyamoto S: The PEST domain of IkappaBalpha is necessary and sufficient for in vitro degradation by mu-calpain. $J$ Biol Chem 1999, 274(43):30874-30881.

19. Oberg C, Li J, Pauley A, Wolf E, Gurney M, Lendahl U: The Notch intracellular domain is ubiquitinated and negatively regulated by the mammalian Sel-10 homolog.J Biol Chem 2001, 276(38):35847-35853.

20. Wu G, Lyapina S, Das I, Li J, Gurney M, Pauley A, Chui I, Deshaies RJ, Kitajewski J: SEL-10 is an inhibitor of notch signaling that targets notch for ubiquitin-mediated protein degradation.Mol Cell Biol 2001, 21(21):7403-7415.

21. Hasselgren PO, Fischer JE: The ubiquitin-proteasome pathway: review of a novel intracellular mechanism of muscle protein breakdown during sepsis and other catabolic conditions.Ann Surg 1997, 225(3):307-316.

22. Hippe A, Bylaite M, Chen M, von Mikecz A, Wolf R, Ruzicka T, Walz M: Expression and tissue distribution of mouse Hax1.Gene 2006, 379:116-126.

23. Grzybowska EA, Sarnowska E, Konopinski R, Wilczynska A, Sarnowski TJ, Siedlecki JA: Identification and expression analysis of alternative splice variants of the rat Hax-1 gene.Gene 2006, 371(1):84-92.

24. Fadeel B, Grzybowska E: HAX-1: a multifunctional protein with emerging roles in human disease.Biochim Biophys Acta 2009, 1790(10):1139-1148.

25. Pickart CM, Fushman D: Polyubiquitin chains: polymeric protein signals. Curr Opin Chem Biol 2004, 8(6):610-616.

26. Hofmann RM, Pickart CM: In vitro assembly and recognition of Lys-63 polyubiquitin chains.J Biol Chem 2001, 276(30):27936-27943.

27. Weissman AM: Themes and variations on ubiquitylation.Nat Rev Mol Cell Biol 2001, 2(3):169-178

28. Lin R, Beauparlant P, Makris C, Meloche S, Hiscott J: Phosphorylation of IkappaBalpha in the C-terminal PEST domain by casein kinase II affects intrinsic protein stability.Mol Cell Biol 1996, 16(4):1401-1409.

29. Marchal C, Haguenauer-Tsapis R, Urban-Grimal D: A PEST-like sequence mediates phosphorylation and efficient ubiquitination of yeast uracil permease.Mol Cell Biol 1998, 18(1):314-321.

30. Lu QL, Poulsom R, Wong L, Hanby AM: BCl-2 expression in adult and embryonic non-haematopoietic tissues.J Pathol 1993, 169(4):431-437.

31. Reed JC: A day in the life of the BCl-2 protein: does the turnover rate of $\mathrm{BCl}-2$ serve as a biological clock for cellular lifespan regulation?Leuk Res 1996, 20(2):109-111.

32. Lee AY, Lee Y, Park YK, Bae KH, Cho S, Lee do H, Park BC, Kang S, Park SG: HS 1-associated protein X-1 is cleaved by caspase-3 during apoptosis. Mol Cells 2008, 25(1):86-90.

33. Han J, Goldstein LA, Hou W, Froelich CJ, Watkins SC, Rabinowich H: Deregulation of mitochondrial membrane potential by mitochondrial insertion of granzyme B and direct Hax-1 cleavage.J Biol Chem 2010, 285(29):22461-22472. 
34. Kasashima K, Ohta E, Kagawa Y, Endo H: Mitochondrial functions and estrogen receptor-dependent nuclear translocation of pleiotropic human prohibitin 2.J Biol Chem 2006, 281(47):36401-36410.

35. Li B, Hu Q, Wang H, Man N, Ren H, Wen L, Nukina N, Fei E, Wang G: Omi/ $\mathrm{HtrA} 2$ is a positive regulator of autophagy that facilitates the degradation of mutant proteins involved in neurodegenerative diseases. Cell Death Differ 2010, 17(11):1773-1784.

36. Ren J, Wen L, Gao X, Jin C, Xue Y, Yao X: DOG 1.0: illustrator of protein domain structures.Cell Res 2009, 19(2):271-273.

doi:10.1186/1471-2121-13-20

Cite this article as: Li et al:: Hax-1 is rapidly degraded by the

proteasome dependent on its PEST sequence. BMC Cell Biology 2012

13:20

\section{Submit your next manuscript to BioMed Central and take full advantage of:}

- Convenient online submission

- Thorough peer review

- No space constraints or color figure charges

- Immediate publication on acceptance

- Inclusion in PubMed, CAS, Scopus and Google Scholar

- Research which is freely available for redistribution 